\title{
The relationship between aesthetic preference and visual complexity in absract art
}

\author{
JOHN W. OSBORNE and FRAVK $H$. \\ FARLEY, University of Wisconsin, \\ Madison. Wis. 53706
}

Ten graduate art students (GAS) and 10 graduate educational psy'cholog.' students (GES), matched for age and sex, rated 62 reproductions of famous paintings in terms of three categories of visual complexity to form a scale of 15 cards extracted from the ratings. Two further groups of 15 matched $S$ s sorted the 15 cards of the risual-complexity scale into a forced distribution of three categories of preference. Results showed a significant relationship between rated visual complexity and aesthetic preference. Both groups preferred high complexit?. No significant sex differences were found for either group. No relationship was found between aesthetic preference and the $E$ scale of the Eysenck Personality Inventory (EPI) or the Sensation Seeking Scale (SSS).

Examination of research on the relation between preference and visual complexity creates a confusing picture. The finding by Vanderplas \& Garvin (1959) that undergraduates associated the most meaning with polygons of low complexity lent credence to the notion that aesthetically preferred forms were simple in structure (Birkhoff, 1933). However, Eysenck (1941) and Barron \& Welsh (1952) found preference to be positively related to increasing complexity, particularly among artists. In addition, Taylor \& Eisenman (1964) found that more creative art students tended to choose more complex figures as preferred and meaningful than did less creative art students.

Some indirect support is available for the notion that moderate levels of complexity may be the most preferred. Eisenman (1966) found that Ss made most associations to random shapes of moderate complexity, while Eisenman \& Rappaport (1967) found that only about one-sixth of their $103 \mathrm{Ss}$ preferred a greater amount of complexity to simplicity. A further complicating variable involved in the relationship between complexity and preference arises from a study by Eisenman (1967) that showed sex to be a highly significant determinant of complexity preference, with females tending to prefer greater complexity.

It is difficult to draw conclusions from such equivocal findings, much less to extrapolate them to the field of abstract art. especially since most of the studies used the 28 figures of Berlyne (1963) or the polygons of Attneave \& Arnoult (1956). The main purpose of this study was to examine the relationship between aesthetic preference and rated visual complexity in abstract art. In addition, Eisenman's finding of significant sex differences in complexity preference was examined.

Barron (1963) has suggested that the person who prefers complexity is more likely to be nonconforming, flexible, and creative than the person who prefers simple stimuli. Consequently, it was hypothesized that Ss who preferred high complexity would have higher extraversion (E) scores on the Eysenck Personality Inventory (EPI) (1964) than those who preferred low complexity. Also, that Ss who preferred high complexity would score higher on the Sensation Seeking Scale (SSS) (Zuckerman, Kolin, Price, \& Zoob, 1964) than those who preferred low complexity.

\section{SUBJECTS}

The Ss were 50 graduate students. Two groups, matched for age and sex, of 10 graduate art students (GAS) and 10 graduate educational psychology students (GES) were used for the initial rating operation. There were five males and five females in each group. For the final aesthetic preference rating, two further matched groups of 15 GAS and 15 GES were used. There were eight males and seven females in each group. The average ages of GAS males and females were 27.9 and 26.4 years, respectively, while the average ages of GES males and females were 28.1 and 26 years, respectively.

\section{STIMULUS MATERIAL}

The stimulus material initially consisted of $62(5 \times 3 \mathrm{in}$.) colored reproductions of abstract paintings by Wassily Kandinsky, Piet Mondrian, Ben Nicholson, and Joan Miro. These artists were chosen on an a priori basis as representing a considerable range of visual complexity. After the initial rating procedure, the number of cards was reduced to form a three-category visual complexity scale of 15 cards that was used in the main stage of the experiment.

PROC'EDURE

The two groups of $10 \mathrm{GAS}$ and $10 \mathrm{GES}$ were asked to sort the 62 cards in to three categories of visual complexity (high, medium, and low). Visual complexity was defined as the way in which the formal elements of line, direction, shape, size, color, tone, and texture were used to produce harmony, contrast, dominance, rhythm, and balance. Stress was put upon the presence of a multiplicity of one or more of these elements to produce visual complexity. The definition was discussed with each $\mathrm{S}$ to avoid misunderstandings. Ss were asked to make their judgments on a first-impression global basis.

Visual complexity ratings for each of the 62 cards were tabulated for both groups. Five cards in each category upon which there was most agreement between the two groups were extracted to form a visual complexity scale. These 15 cards were presented to the two further groups of 15 GAS and 15 GES, who were asked to sort them into a forced distribution of three categories of aesthetic preference. Finally, Ss completed the SSS and the E scale of the EPI.

\section{RESULTS}

The mean preference scores of GAS and GES are presented in Table 1. Preference scores were based on a weighted scoring system of 3, 2, 1 for first, second, and third preferences, respectively. As Table 1 shows, there were no significant sex differences in aesthetic preference scores. The same trend of preference for visual complexity appeared in both GAS and GES groups.

Figure 1 shows graphically the trends evident in Table 1 regarding the relationship between aesthetic preference and visual complexity. For both GAS and GES, the relationship was positive, linear, and decelerating. A Friedman two-way analysis of variance by ranks resulted in the rejection of the null hypothesis of no significant difference in the sums of the preference ranks for each category of complexity for both groups (GAS, $\chi^{2}(2)=6.93, p<.05 ; \operatorname{GES}, \chi^{2}(2)=6.53$ $\mathrm{p}<.05)$.

The scores of Ss preferring high or low visual complexity in both GAS and GES groups were not significantly correlated

Table 1

Mean Aesthetic Preference Scores

\begin{tabular}{|c|c|c|c|c|c|c|}
\hline \multirow[b]{2}{*}{ Complexity } & \multicolumn{3}{|c|}{$\begin{array}{l}\text { Graduate Educational } \\
\text { Psychology Students }\end{array}$} & \multicolumn{3}{|c|}{ Graduate Art Students } \\
\hline & Male & Female & Combined & Male & Female & Combined \\
\hline High & 11.87 & 11.14 & 11.50 & 12.62 & 11.14 & 11.88 \\
\hline Medium & 11.87 & 10.57 & 11.22 & 9.87 & 10.85 & 10.35 \\
\hline Low & 7.37 & 8.28 & 7.82 & 7.5 & 8.0 & 7.75 \\
\hline
\end{tabular}




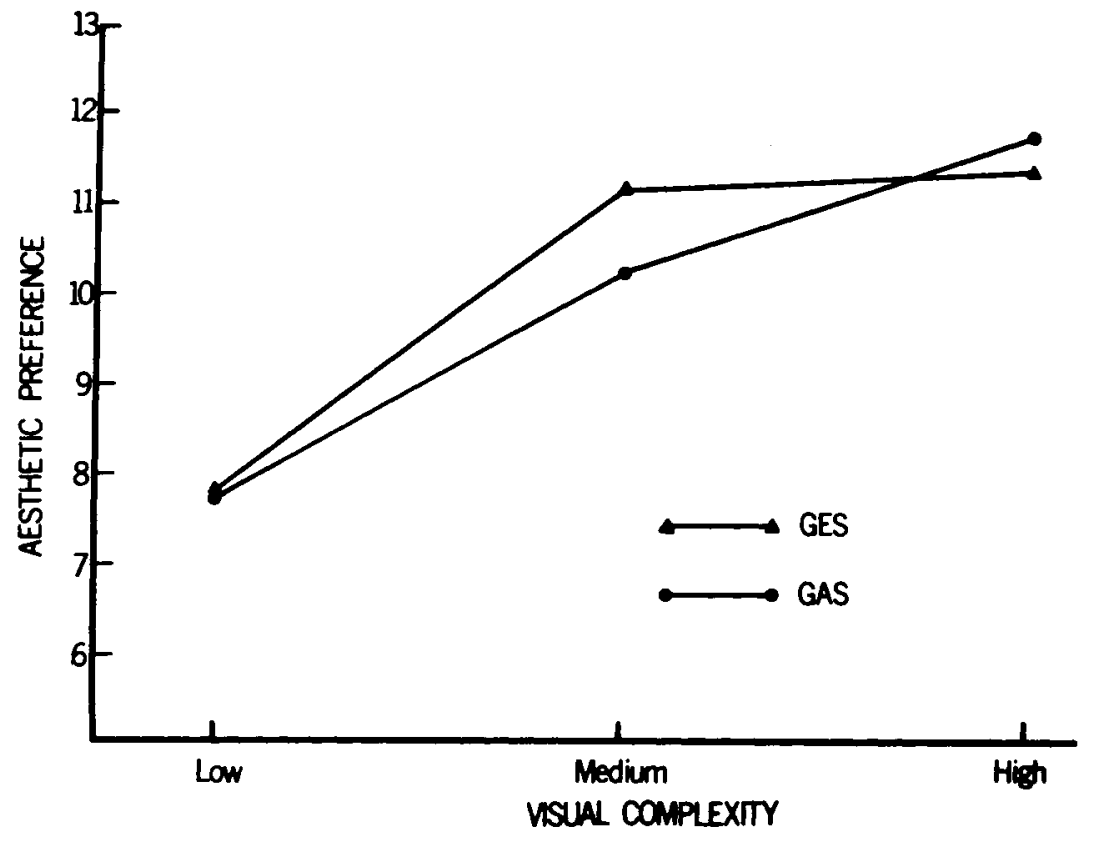

with scores on the SSS or the E scale of the EPI. The scores of all Ss showed little variance on these two measures.

$$
\text { DISCUSSION }
$$

The main purpose of this study was to investigate the relationship between rated visual complexity and aesthetic preference with actual works of art rather than experimentally derived stimuli such as those of Attneave and Arnoult or Berlyne. The problem in doing this is the lack of control over the stimulus. However, it was felt that there would be a considerable amount of validity in a complexity scale that was determined by the responses of Ss to the initial collection of 62 cards. The limitation of this approach is that the scale is relative to the two groups of Ss, as must be the findings of the study. An applied experiment such as this faces the problem of multiple uncontrolled variables in the stimulus material.

The works of art chosen were abstract in order to avoid realism that might interfere with judgment of complexity. The work of Kandinsky was judged by the Es to be the most visually complex on an a priori basis, and this is, in fact, the way Ss judged it in the initial rating study. Some of the works of Nicholson, Mondrian, and Miro were judged by the Es to be the least visually complex and were also rated as such with
Fig. 1. The relationship between aesthetic preference and visual complexity.

preference and $\mathrm{E}$ or the SSS may have been an artifact of the restrictive sampling procedures used in the study. Similar experiments with larger heterogeneous samples may reveal relationships between aesthetic preference and personality.

The use of GAS and GES as Ss was designed to detect the possibility of a factor unique to either group's aesthetic preferences. The similarity between the groups may indicate that the basis for aesthetic preference has at least limited generality.

\section{REFERENCES}

ATTNEAVE, F., \& ARNOULT, M. D. Methodological considerations in the quantitative study of shape and pattern perception. Psychological Bulletin. 1956.53. 452-471.

BARRON, F. Creativity and psychological health. Princeton, N.J: Van Nostrand, 1963.

BARRON, F., \& WELSH, G. S. Artistic perception as a possible factor in personality style: Its measurement by a figure preference test. Joumal of Psychology, 1952, 33, 199-203.

BERLYNE, D. E. Complexity and incongruity variables as determinants of exploratory choice and evaluative ratings. Canadian Journal of Psychology, 1963, 17, 3, 274-290.

BIRKHOFF, G. D. Aesthetic measure. Cambridge: Harvard University Press, 1933.

EYSENCK, H. J. The empirical determination of an aesthetic formula. Psychological Review. $1941,48,83-92$.

EYSENCK, H. J., \& EYSENCK, S. B. G. The Eysenck Personality Inventory. San Diego, Calif: Education and Industrial Testing Service, 1964.

EISENMAN, R. The association of random shapes revisited. Psychonomic Science, 1966, 6, 397-398.

EISENMAN, R. Complexity-simplicity: II. Birth order and sex differences. Psychonomic Science, 1967, 8, 171-172.

EISENMAN, R., \& RAPPAPORT, J. Complexity preference and semantic differential ratings of complexity-simplicity and symmetry-asymmetry. Psychonomic Science, $1967,7,147-148$.

TAYLOR, R. E., \& EISENMAN, R. Perception and production of complexity by creative art students. Journal of Psychology, 1964, 57, 239-242.

VANDERPLAS, J. M., \& GARVIN, E. A. The association value of random shapes. Journal of Experimental Psychology, 1959, 57, 147-154.

ZUKERMAN, M., KOLIN, E. A., PRICE, L., \& ZOOB, I. Development of a sensation seeking scale. Journal of Consulting Psychology, 1964 , $28,477-482$. 